\title{
Pushing the limits - latest developments in angle metrology for the inspection of ultra-precise synchrotron optics
}

\author{
Tanfer Yandayan*a, Ralf D. Geckeler ${ }^{\mathrm{b}}$, Frank Siewert ${ }^{\mathrm{c}}$ \\ ${ }^{a}$ TUBITAK Ulusal Metroloji Enstitüsü, Dr.Zeki Acar Cad. No:1, 41470 Gebze-Kocaeli, Turkey; \\ ${ }^{\mathrm{b}}$ Physikalisch-Technische Bundesanstalt, Bundesallee 100, 38116 Braunschweig, Germany; \\ ${ }^{\mathrm{c}}$ Helmholtz Zentrum Berlin, Institut für Nanometer Optik und Technologie, Albert-Einstein-Str. 15, \\ 12489 Berlin, Germany
}

\begin{abstract}
The requirements on the quality of ultra-precise X-ray optical components for application in the Synchrotron Radiation (SR) community are increasing continually and strongly depend on the quality of the metrology devices available to measure such optics. To meet the upcoming accuracy goal of $50 \mathrm{nrad}$ rms for slope measuring profilers, a dedicated project, SIB58 Angles, consisting of 16 worldwide partners and supported by the European Metrology Research Programme (EMRP) was started in Sep 2013. The project covers investigations on autocollimators under extremely challenging measuring conditions, ray-tracing models, 2D autocollimator calibration (for the first time worldwide), determination of error sources in angle encoders providing traceability by 'sub-division of $2 \pi$ rad' with nrad uncertainty, angle generation by 'ratio of two lengths' in nrad level, and on the development of portable precise Small Angle Generators (SAGs) for regular in-situ checks of autocollimators' performance. Highlights from the project will be reported in the paper and the community of metrology for X-Ray and EUV Optics will be informed about its progress and the latest work in angle metrology.
\end{abstract}

Keywords: Angle metrology, autocollimators, profilometers, SR optics, SI unit radian, X-Ray and EUV Optics, XFELoptics, form measurement

\section{INTRODUCTION}

Angle metrology is a key technology for scientific and industrial applications of high value, enabling countries to be globally competitive. Precise angle measuring devices - such as angle encoders, angle interferometers, small angle generators and autocollimators - are extensively used in various applications where high precision is demanded and are calibrated by national metrology institutes (NMIs) in order to ensure the traceability to SI unit of plane angle, radian (rad) which is defined as ratio of two lengths [1]. The SI unit of plane angle, radian, derived from the length unit as in the definition may also be realized (even more practically) by subdivision of a full circle, $2 \pi$ rad [2-15]. The NMIs may use both methods for ensuring the traceability and mostly calibrate polygon mirrors, angle encoders and autocollimators for industry, research organizations and universities.

Assurance of traceability by demonstrating equivalence between national measurement standards on global level to the SI for the base units and derived units (such as radian) has been carried out within the international metrology system created by the Metre Convention (1875) that established a permanent organizational structure to act on all matters relating to units of measurement [16]. Today, metrology is a real international venture with 56 states being members of the convention, including all major industrialised countries. The National Metrology Institutes (NMIs) are the core part of the system and have being working jointly within the Regional Metrology Organisations (RMOs) towards the international harmonisation of metrology with primary concerns of mutual recognition activities, intercomparison measurements, traceability, training and consultancy. RMO of Europe, EURAMET e.V [17] is also responsible for the elaboration and execution of the European Metrology Research Programme (EMRP) which is designed to encourage collaboration between European NMIs and partners in industry or academia [18]. The programme, jointly funded by the EMRP participating countries within EURAMET and the European Union funds joint research projects in specific fields of metrology $[17,18]$.

*tanfer.yandayan@tubitak.gov.tr; phone 90262679 5000; fax 90262679 5001; www.ume.tubitak.com.tr 
European NMIs have taken new demands of advanced angle metrology into account and proposed a Joint Research Project (JRP) under EMRP with the support of angle metrology equipment manufacturers and users (e.g. Research organisations working on X-Ray and EUV Optics, XFEL-optics). The proposed project, SIB58 Angles - Angle metrology, was accepted and will run in September 2013-2016 to improve the dissemination of the SI angle unit 'radian' which is currently in demand by the most challenging applications [19, 20]. Specifically, it aims to enable traceability of the measurand 'angle' with lower uncertainty (less than 50 nrad).

The consortium of SIB58 Angles project, consisting of 16 worldwide partners, brings together the leading ten European NMIs (TUBITAK, CEM, CMI, INRIM, IPQ, LNE, MG, MIKES, PTB and SMD) in high-accuracy angle metrology; they are complemented by one research institute having high level experience in precision engineering and manufacturing (IK4-TEKNIKER), two non-European leading NMIs (AIST and KRISS) in angle metrology and two companies that bring in the specific knowledge and experience in autocollimators (MWO) and angle encoders (FAGOR AUTOMATION) $[19,20]$. All of them are supported by one of the world leading experienced research organisation on X-Ray and EUV Optics, XFEL-optics from synchrotron community (HZB) under special scheme of EMRP, called Researcher Excellence Grant (REG) [18-20]. This provides substantial contribution for guiding the research activities in the project considering the needs for improvement of SR optics. In addition, the project is in progress to have further cooperations with its stakeholders in the schemes of 'Collaborator' and 'Stakeholder Committee member'. This will improve the research activities carried out in the project and will produce high level impact.

This paper will give a summary and information on first results of the project SIB58 Angles. After explaining recent developments and the demands in angle metrology related to inspection for the quality of synchrotron radiation (SR) optics, the activities carried out for the use and realisation of the SI unit radian will be reported. An overview of the first precise calibration of autocollimators with angle encoders by use of a shearing technique (achieving the Separation of autocollimator and angle encoder errors at an uncertainty level of 1 milliarcsec $(5 \mathrm{nrad})$ ) will be shown. Establishment of cooperative work between manufacturers, research organisations and the users for solving of one of the important problems during investigation of SR optics (a novel device for reproducible aperture alignment relative to autocollimator's optical axis in the level of $0.1 \mathrm{~mm}$ ) and its progress will be presented. The specific needs of the end users, e.g. current and also future needs of accelerator based (ring- and linac-type) synchrotron facilities, determined for calibration of autocollimators used in challenging conditions will be illustrated. The progress work on investigations carried out on autocollimators under extremely challenging measuring conditions, ray-tracing models, 2D autocollimator calibration (for the first time worldwide), determination of error sources in angle encoders providing traceability by 'subdivision of $2 \pi$ rad' with nrad uncertainty, angle generation by 'ratio of two lengths' in nrad level, and on the development of portable precise Small Angle Generators (SAGs) for regular in-situ checks of autocollimators' performance will be presented. Highlights from the recent developments in advanced angle metrology reported in the paper will provide a bridge between angle metrology community of National Metrology Institutes, manufacturers and the community of metrology for X-Ray and EUV Optics.

\section{ADVANCED ANGLE METROLOGY FOR INSPECTING THE QUALITY OF X-RAY OPTICS}

\subsection{Slope measuring instruments in use for the inspection of synchrotron optical elements}

Since the late 1980s the use of slope measuring instruments became a standard technique for the inspection and characterization of optical elements to be used under grazing incidence in synchrotron application [21, 22, 23] and astronomy [24]. Instruments like the Long Trace Profiler (LTP) [23, 25], the Deflectometric Flatness Reference (DFR) $[26,27,28]$ or the Nanometer Optical Component Measuring Machine [29, 30] enable the inspection of reflective free form surfaces by a direct measurement of the deflection angle of a laser beam. The classical LTP-II as provided first by Continental Optics Ltd. was enabling an accuracy of $0.5 \mu \mathrm{rad} \mathrm{rms}$ for a slope measurement. Due to the increasing demands for high quality optical elements and thus the accuracy of metrology devices, many laboratories have made upgrades of the existing LTP's [31, 32] or have developed new instruments. In this context the moving penta-prism concept was realized at several labs $[33,34,35,36]$ and has shown significant advantages. It was shown that some of these instruments enable the inspection of optics with $0.05 \mu \mathrm{rad}$ rms accuracy $[32,34,36]$. An alternative concept is the Nanometer Optical Component Measuring Machine NOM, designed at BESSY [29, 30]. The NOM is a hybrid of a LTPhead, designed by Peter Takacs and an autocollimator made by Möller Wedel Optical GmbH. Enabling an accuracy of $0.05 \mu \mathrm{rad} \mathrm{rms}$ for plane and slightly curved optics the BESSY-NOM represents a 2nd generation slope measuring profiler [26, 27, 28, 37]. Further NOM or NOM-like instruments have been commissioned recently at Diamond Light Source (UK) [38], ALBA Synchrotron (Spain) [39], APS-Advanced Photon Source (USA) [40, 41], NSLS-II (USA) [42] 
and at the LBNL / ALS - Optics and Metrology Lab in Berkeley (USA) [43]. These highly accurate instrumentation are essential for a detailed characterization of upcoming high performance reflective optical elements like extreme long super-plane mirrors for FEL application [44], diffraction limited focusing optics [45] or super-polished substrates for multilayer-monochromators [46, 47].

Systems like the Long Trace Profiler (LTP) or an autocollimator as used at the NOM enable the inspection of reflective surfaces by a direct measurement of the deflection angle of a laser beam. In contrast to interferometry these instruments do not rely on external references. A laser source generated test beam is traced over the sample along the line of inspection. Depending on the local topography the test beam will be reflected into the position sensitive detector of the sensor head. The position of the reflected test beam on the CCD-line of the sensor is directly related to the local surface slope, see Figure 1. The reflection of the test beam along the optical axis of the instrument is determined by the angle between the mirror normal and the direction of the impinging laser beam $[48,49,50]$. The relative slope change is measured by scanning along the line of inspection. The sensor detects the change of the angle of reflection from one position $\mathrm{x}$ on the substrate to the next position $\mathrm{x}+\Delta \mathrm{x}$.. Figure 1 shows the optical setup for the scanning penta-prism configuration by use of an autocollimator as sensor at the NOM. Than, the topography profile $\mathrm{h}(\mathrm{xk})$ is extracted by spatial integration of the slope data. Applying improved integration algorithms such as spline interpolation became an option to handle more noisy data as well [51]. The residual figure error is gained by subtraction of an ideal profile e.g. a circle or an elliptical fit. Optical systems up to a length of $1600 \mathrm{~mm}$ in length can be aligned for inspection at the NOM. The maximum possible scan length of the instrument is $1200 \mathrm{~mm}$. The spatial period range covered by NOM is from $2 \mathrm{~mm}$ to the aperture length. Virtually any curved, reflective optical shape can be measured as long as the slope change is within a $\pm 5 \mathrm{mrad}$ acceptance angle. Stitching techniques can be applied in case a long optical surface of significant curvature needs to be measured. It was shown recently that a careful characterization and calibration of the sensor in use is essential to achieve the required measurement performance [37, 51, 52, 53].

Apart from slope measurement methods and flatness interferometry, other methods also exist in the literature for optical measurements of large surfaces. In such methods, optical displacement measurements are carried out using the displacement interferometer configurations [54, 55].

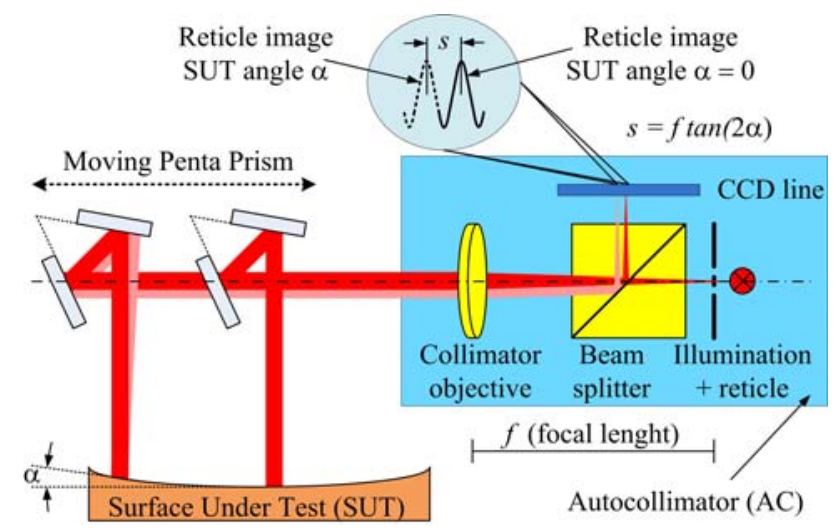

Figure 1. Optical lay out of a moving penta-prism slope measuring profiler

\subsection{Autocollimators and calibration for traceability}

Autocollimators are optical instruments which measure angular displacement of reflecting surfaces. They have a wide range of applications in metrology and industrial manufacturing such as calibration of angular standards (polygons, angle gauge blocks, indexing tables etc.), measuring straightness, parallelism, rectangularity of machine tools and coordinate measuring machines, flatness of surface plates and checking of optical parts and components etc. [2, 56, 57, 58].

As any other measuring instrument, autocollimators must be calibrated to detect and correct any systematic error and determine the uncertainty associated to their readings, making their measurements traceable to the SI plane angle unit. Calibration of autocollimators with full aperture size will be sufficient for use of the autocollimators in classical applications such as calibration of angular standards (polygons, angle gauge blocks, indexing tables etc.). However, use of autocollimators for the ultra-precise measurement of optical surfaces using scanning techniques (such as inspecting 
the quality of SR optics) requires further investigations and more detailed calibration to obtain better accuracies (than those stated by the manufacturers). This is only possible after individual calibration of the devices customised to the specific measuring tasks.

The calibration of autocollimators is conveniently carried out by the use of so-called small angle generators (sine or tangent arm) in which the angle measurement is traceable to the length unit (ratio of two lengths) [2, 59]. Although, expanded uncertainties of 0.01 arcsec is achieved, the measurement ranges are usually small such as 30 arcsec [56, 5962]. Use of rotary tables fitted with angle encoders has advantages for calibration of autocollimators particularly in the large ranges. However this requires careful inspection of the system and calibration. In general, low uncertainties for generation of small angles (e.g. less than 0.05 arcsec) are achieved with multiple reading head special angle encoders [8, $11,12-14]$. Use of single reading head commercial angle encoders posses problems and they can only be used for demanded low uncertainties if such problems are eliminated with special procedures and strategies [57].

The current official capabilities of the NMIs for calibration of autocollimators can be obtained from the Calibration Measurement Capabilities (CMC) database of the International Bureau of Weights and Measures (BIPM) [60]. According to the current CMC data, calibration of autocollimators with the uncertainty values and measurement ranges have recently been listed in a chart with the utilised methods [57]. Various techniques are used for calibration of autocollimators ranging from use of rotary tables fitted with angular encoders (multiple/single reading head) to small angle generators and angular interferometers. The lowest uncertainty $(\mathrm{U}=0.01 \mathrm{arcsec})$ is achieved by special angle comparator of PTB (NMI of Germany) in clean room at a highly constant ambient temperature $(\Delta \mathrm{T}<0.05 \mathrm{~K})$ and with a continuous laminar airflow $(\mathrm{v}=20 \mathrm{~cm} \mathrm{~s}-1)[11,60]$.

First international intercomparison measurements for calibration of high-resolution electronic autocollimators were started in 2009 (EURAMET.L-K3a.2009) to provide information on the capabilities and limits of independent calibration methods and devices [63]. The output of this will give objective information for better evaluation of the uncertainty values in the CMC list.

\subsection{Demands for inspection of ultra-precise synchrotron radiation (SR) optics}

Future application of slope measuring profiler will become more challenging. The inspection of long ultra-precise slightly curved focusing mirrors up to a length of $1000 \mathrm{~mm}$ with design parameter for the residual slope deviation in the range of $50 \mathrm{nrad} \mathrm{rms}$ is under discussion [64]. To measure these mirrors an angular range of about $\pm 50 \mu \mathrm{rad}$ on the detector is needed to cover the full slope profile of the optics.

In addition, strong curved mirrors with significant curvature are required to focus UV- and VUV-light. In case of these elliptical cylinder mirrors a change of the local curvature is expected from $15 \mathrm{~m}$ to $5 \mathrm{~m}$. The required slope error is of about $0.2 \mu \mathrm{rad} \mathrm{rms}$ on a length of $200 \mathrm{~mm}$ to $300 \mathrm{~mm}$. To measure such mirrors an angular range of $\pm 20 \mathrm{mrad}$ would be required. However, it should also be noted that state of the art autocollimators used at slope measuring profilers like NOM or ESAD cover an angular range of $\pm 5 \mathrm{mrad}$.

The above mentioned parameters used during inspection of SR optics are schematically illustrated in Figure 2.

Mostly mirrors are measured in face up-condition but there is a strong demand to measure such optical components in the state as they will be installed finally. Thus measurements in face-side as well as in face-down condition including the mounted state are required [65].

Measurement of mirrors in face-up (vertical orientation) and face-side (horizontal orientation) are illustrated in Figure 3 and Table 1 summarizes the demands for inspection of ultra-precise synchrotron radiation (SR) optics.

New generation of highly accurate angle-measuring optical profilometers have to provide single nm-accuracy on a length scale of $1000 \mathrm{~mm}$ not only for flat surfaces but also for curved apertures. These optics need to be measured with comparable accuracy. A second topic under discussion is the spatial resolution to be achieved with autocollimator based slope measuring profiler. Still a spatial resolution of 1 to $2 \mathrm{~mm}$ (depending on the diaphragm size used to shape the measurement beam) is state of the art. An improvement to a value of $0.5 \mathrm{~mm}$ would be a significant step forward. It is noted here that of course interferometric methods provide significant higher resolution but show limitations in case of curved optics as well as for super-flat optics (radii $>100 \mathrm{~km}$ ). Thus future work on autocollimators for application in slope measuring profiler should address such topics like small sized measurement beam and a high angular resolution with a robust performance of the instrument along an acceptable large angular range (e.g. +/- 2000" would be nice, but even $+/-500$ " is an acceptable compromise). 


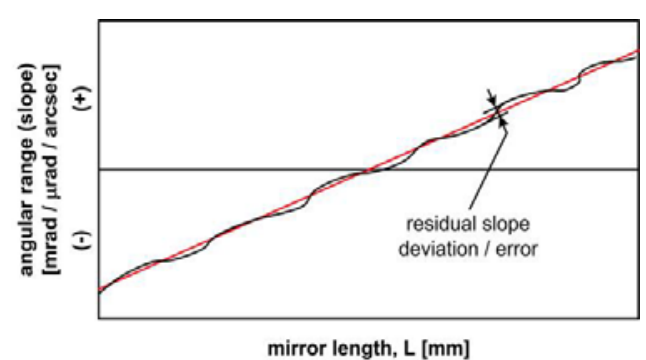

(a) Angular data taken by Autocollimator

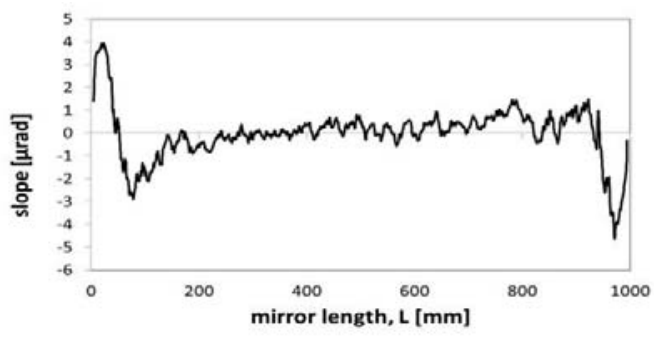

(c) Residual slope

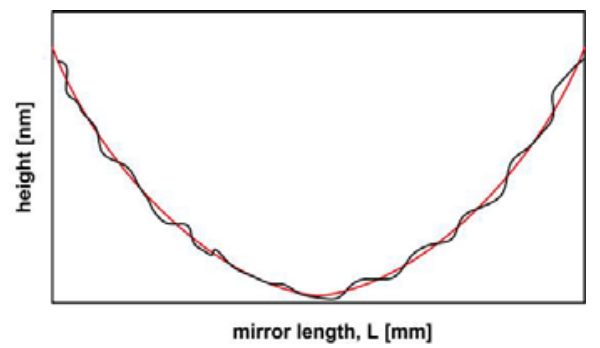

(b) Figure of the mirror gained by integration of the slope data

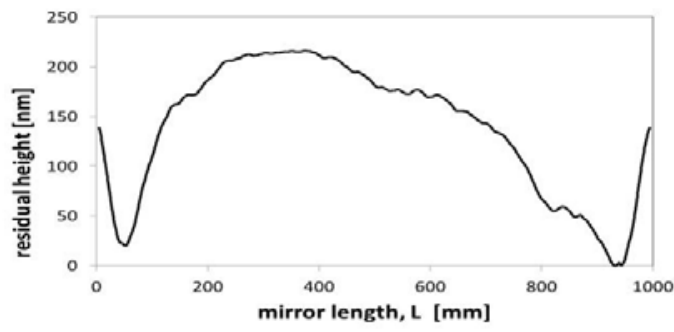

(d) Residual height

Figure 2. Illustration of parameters used during inspection of SR optics: (a) and (b) illustrate the angular data and corresponding height values as an example. (c) and (d) show the profiles of residual slope and height on a toroidal mirror to collimate synchrotron light at a beamline at the BESSY-II storage ring, the aperture section is from mirror position x 200 $800 \mathrm{~mm}$

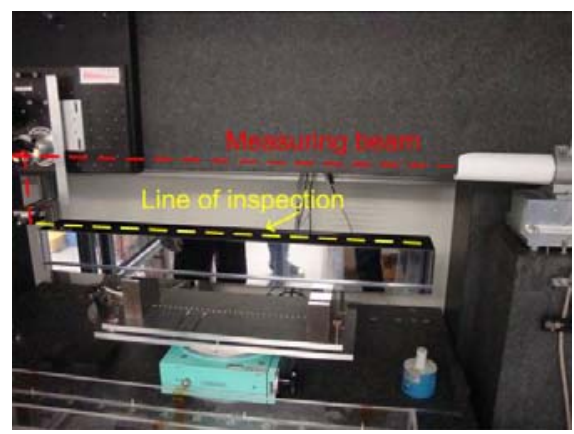

(a) Use of autocollimator in VERTICAL orientation

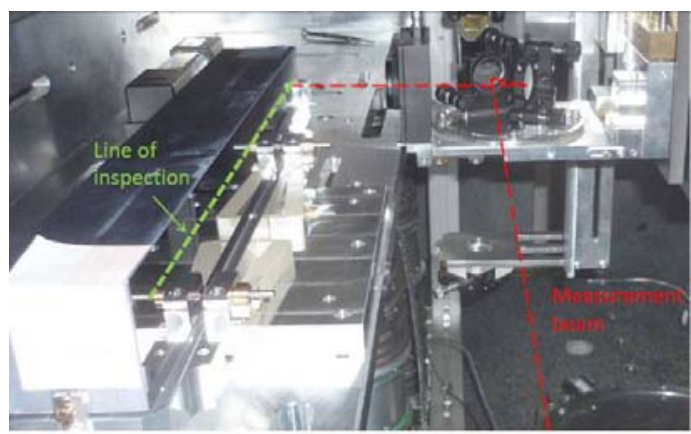

(b) Use of autocollimator in HORIZONTAL orientation

Figure 3. Different synchrotron optical components under inspection at the BESSY-NOM: (a) A $800 \mathrm{~mm}$ long beam distribution mirror for FLASH at DESY in face-up condition (b) An elliptical cylinder shaped horizontal focusing mirror for LCLS (Stanford) in face side condition

Table 1. Summary of requirements for inspection of ultra-precise SR optics

\begin{tabular}{|l|l|l|l|l|}
\hline $\begin{array}{c}\text { Mirror Length } \\
\text { (L: mm) }\end{array}$ & $\begin{array}{c}\text { Residual Slope } \\
\text { Deviation / Error (rms) }\end{array}$ & $\begin{array}{c}\text { Required } \\
\text { Angular Range }\end{array}$ & $\begin{array}{l}\text { Measurement } \\
\text { Condition }\end{array}$ & \multicolumn{1}{|c|}{ Remarks } \\
\hline $30-1000$ & $\begin{array}{l}\leq 50 \mathrm{nrad} \\
(\leq 0.01 ”)\end{array}$ & $\begin{array}{l} \pm 50 \mu \mathrm{rad} \\
\left( \pm 10^{\prime \prime}\right)\end{array}$ & $\begin{array}{l}\text { Face-up } \\
\text { Face-side } \\
\text { Face-down }\end{array}$ & $\begin{array}{l}\text { Long ultra-precise slightly } \\
\text { curved focusing mirrors. }\end{array}$ \\
\hline $100-300$ & $\begin{array}{l}\leq 0.2 \mu \mathrm{rad} \\
(\leq 0.04 ”)\end{array}$ & $\begin{array}{l} \pm 20 \mathrm{mrad} \\
\left( \pm 4125^{\prime \prime}\right)\end{array}$ & $\begin{array}{l}\text { Face-up } \\
\text { Face-side } \\
\text { Face-down }\end{array}$ & $\begin{array}{l}\text { Strong curved mirrors with } \\
\text { significant curvature to } \\
\text { focus UV- and VUV-light. }\end{array}$ \\
\hline
\end{tabular}




\section{CALIBRATION OF AUTOCOLLIMATORS USED IN OPTICAL PROFILOMETRY}

\subsection{Features and parameters of instrument for calibration of autocollimators used in optical profilometry}

Requirements for future synchrotron optics described in Section 2 define the performance parameters of the calibrator that will be used for calibration of the autocollimators particularly used in profilometers.

These calibration tools should be able calibrate the autocollimators in long measurement distances to the reference mirror (such as $200 \mathrm{~mm}-1200 \mathrm{~mm}$ ) for a small angular range of about $\pm 50 \mu \mathrm{rad}( \pm 10$ ”) with an expanded uncertainty better than $50 \mathrm{nrad}(0.01$ ") and high angular resolution such as $1 \mathrm{nrad}(0.0002$ "). The last mentioned requirement should be subject of upcoming investigations on how realistic these goals are, mainly with respect to large measurement distances.

On the other hand such an instrument should allow us to calibrate the autocollimator in short measurement distances to the reference mirror (with a distance variation of $200 \mathrm{~mm}-500 \mathrm{~mm}$ ) for large angular ranges such as $\pm 20 \mathrm{mrad}( \pm$ 4125 ") with expanded uncertainties of $0.2 \mu \mathrm{rad}$ (0.04") for the required angle generation using the recommended resolutions of about $10 \mathrm{nrad}(0.002 ")$.

These requirements are challenging to be realized within one single device. Thus the development of two different types of calibration tools for very small angular ranges on long measurement distances and secondly for large angular ranges on shorter measurement distances could be an acceptable compromise. But one must remember the fact that the main challenge here is to cover the vastly different measurement ranges such as $\pm 50 \mu \mathrm{rad}\left( \pm 10^{\prime \prime}\right)$ and $\pm 20 \mathrm{mrad}$ ( \pm 4125 ") separated by a factor of 400 rather than the different distances between autocollimator and reference mirror. Besides, as there is no high resolution autocollimator available in such large range, the priority has to be given for the currently used autocollimator's ranges, $\pm 5 \mathrm{mrad}( \pm 1031 ")$.

An in-situ calibration of the angle detector as installed at the profiler under face-up, face side and face-down condition is essential. Furthermore these devices should allow a simple handling. Many laboratories use e.g. the Long Trace Profiler for the inspection of SR optics. A universal tool for calibration of the angle detectors should also enable the calibration of such instruments as well (usability for angle-measuring devices in general). These tools should work under different laboratory conditions (e.g. clean-room-environment, classical metrology lab environment) also an easy handling and data read out is a must.

It is noted here that several approaches to develop such calibration tool are known from the last few years like the universal test mirror (UTM) [52] and the vertical angle comparator VAT [37] see Figure 4. Both systems were developed at synchrotron labs and are expert systems. The handling of these devices is in part complicated and time consuming. Their accuracy is in the range of (50-100) nrad rms [37]. A further non-technical, but nevertheless important issue is the question of affordable costs to buy such a device. Table 2 summarises features and parameters of the instrument (calibrator) to be used in the metrology departments of accelerator based (ring- and linac-type) synchrotron facilities.

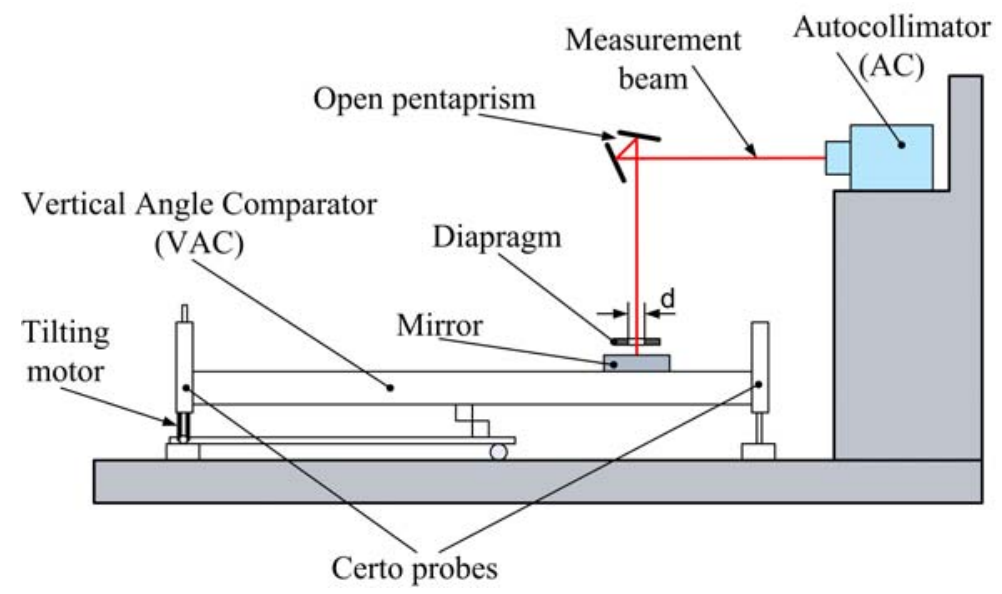

Figure 4. Schematic view for the Vertical Angle Comparator (VAC) at the BESSY-II Optics Lab 
Table 2. Features and parameters of the instrument for calibration of autocollimators (ACs) used in profilometry.

\begin{tabular}{|l|l|l|l|l|}
\hline $\begin{array}{c}\text { Distance between Ref. } \\
\text { Mirror and AC (D: mm) }\end{array}$ & \multicolumn{1}{|c|}{$\begin{array}{c}\text { Required Exp. } \\
\text { Uncert. U (k=2) }\end{array}$} & \multicolumn{1}{|c|}{$\begin{array}{c}\text { Required } \\
\text { Angular Range }\end{array}$} & $\begin{array}{l}\text { Measurement } \\
\text { Condition }\end{array}$ & \multicolumn{1}{|c|}{ Remarks } \\
\hline $200-1200$ & $\begin{array}{l}\leq 50 \mathrm{nrad} \\
(\leq 0.01 ")\end{array}$ & $\begin{array}{l} \pm 50 \mu \mathrm{rad} \\
( \pm 10 ")\end{array}$ & $\begin{array}{l}\text { Face-up } \\
\text { Face-side } \\
\text { Face-down }\end{array}$ & $\begin{array}{l}\text { Recommended } \\
\text { resolution } \\
1 \text { nrad (0.0002") }\end{array}$ \\
\hline $200-500$ & $\begin{array}{l}\leq 0.2 \mu \mathrm{rad} \\
(\leq 0.04 ”)\end{array}$ & $\begin{array}{l} \pm 5 \mathrm{mrad} \text { or to } \pm 20 \mathrm{mrad} \\
( \pm 1031 " \text { or to } \pm 4125 ")\end{array}$ & $\begin{array}{l}\text { Face-up } \\
\text { Face-side } \\
\text { Face-down }\end{array}$ & $\begin{array}{l}\text { Recommended } \\
\text { resolution } \\
10 \text { nrad (0.002") }\end{array}$ \\
\hline
\end{tabular}

\subsection{Further requirements and investigations in calibration of autocollimators used for inspecting the quality of synchrotron radiation (SR) optics}

Application of autocollimators in profilometry requires more specific calibration of these devices [66,67]. Due to special use of autocollimators in profilometers, it is also advisable to check the behaviour of both autocollimator axes (X and Y) since the crosstalk effect was observed in many autocollimators, when the axis values are used in extreme ranges (e.g. when $\mathrm{X}$ axis value is used with high $\mathrm{Y}$ axis values).

Autocollimators may be calibrated in situ in order to have the autocollimator under the same operating conditions as in the specific measurement application, so getting a common behaviour and avoiding to consider different influences and sources of uncertainty, making more realistic the corrections to be applied and the uncertainties associated to such corrections.

In the special case of slope measuring profiler the $\mathrm{Y}$ axis is mainly used (in $90 \%$ of all cases). But applications for faceside characterization of optical components required the $\mathrm{X}$ axis to be used with the same level of accuracy. Hence the calibrator should provide to be applied for large distance variations from autocollimator-aperture to measurement position and should provide to be used for both the $\mathrm{X}$ and the $\mathrm{Y}$ axis.

In project SIB58 Angles, different calibrators so called Large Range Small Angle Generators (LRSAGs) utilising the radian definition - the ratio of two lengths are in the process of development. Additionally, investigations for improvement of angle encoders for realisation of radian in high precision level using circle division methods and investigations to characterise the behaviour of the autocollimators used in profilometry are in progress. These are summarized below.

\section{IMPROVING THE PERFORMANCE OF AUTOCOLLIMATORS}

The aim of the Work Packages 'Metrological characterisation of autocollimators' and 'Application of autocollimators in profilometry' of project SIB58 Angles is to significantly improve angle metrology with existing commercial autocollimators. The focus will be on improving their performance under extremely challenging measuring conditions, such as small (mm-sized) apertures, a variable path length of the measuring beam, curved reflecting surfaces, and beam deflections in both orthogonal measuring axes. These measuring conditions are highly relevant to the application of autocollimators in deflectometric profilometers for the precision form measurement of beam-shaping optical surfaces for synchrotron and FEL applications. The results aim at creating significant advances in the form measurement of that optics which relies on autocollimator-based surface profilometers. The limits of the angle measurement with the autocollimator directly define the limits of the form measurement. Ultimately, these limits define the manufacturing limits of beam-shaping optics by advanced surface modification technologies.

\subsection{Metrological characterisation of autocollimators}

The angle response of an autocollimator is strongly affected by its measuring conditions. This Work Package focuses on the influence of the path length of the autocollimator beam, of the sagittal beam deflection by the Surface Under Test (SUT), and of small apertures for restricting the autocollimator beam which cause diffraction and interference. More details on this topic are provided in a review of the state of the art of autocollimator application, performance, and calibration which can be accessed via [20]. 
One focus is on investigating the influence of the optical path length of the autocollimator beam on the autocollimator's angular response $[68,69]$. This is essential for deflectometric profilometers as most of them use a moving pentaprism to scan the SUT, which induces large (in the range of 1-2 m) and unavoidable changes in this parameter. Due to the varying optical path length, the reflected beam follows different geometrical paths through the autocollimators' optics and, in conjunction with aberrations and alignment errors of the optical components, path-length-dependent angle measurement deviations are induced. As part of this investigation, we will perform extensive experimental characterisation and calibration of autocollimators with varying beam path lengths. Additionally, ray tracing models of autocollimators will be deployed to link experimental data to opto-mechanical causes. This work is in progress.

Another focus is on enabling two-axis (2D) calibrations of autocollimators for the first time. In most autocollimator applications, the autocollimator beam is deflected in two orthogonal angular directions by the SUT. The simultaneous engagement of both measuring axes results in crosstalk between them; i.e., their angle measurements are not independent of each other. Reasons for this include alignment errors and optical aberrations of the autocollimator's internal components, and imperfections of the reticles which are imaged onto the autocollimator's CCD. To achieve these goals, two different set-ups for 2D autocollimator calibrations will be realised.

At the Physikalisch-Technische Bundesanstalt (PTB), Germany, the Spatial Angle Autocollimator Calibrator (SAAC) has been set up and is currently being commissioned. It makes use of an innovative Cartesian arrangement of three autocollimators, two reference autocollimators and the autocollimator to be calibrated, all orthogonal with respect to each other [70]. At the Finnish Centre for Metrology and Accreditation (Mittatekniikan Keskus Mättekikcentralen MIKES), a system based on an independent angle measurement method, angle interferometry, will be realised. It is currently being designed. Both set-ups will complement each other due to their differing physical principles of spatial angle measurement (autocollimators vs. angle interferometry).

As part of both projects, several important problems need to be solved, such as the determination of geometrical adjustment parameters from a suitable set of calibration measurements and the subsequent use of these parameters for correcting the angle measurements by means of special software. A raytracing model of PTB's SAAC has already been created [71].

We also aim to improve autocollimator performance at small (mm-sized) apertures which is of special importance for achieving improved lateral resolution with autocollimator-based deflectometric profilometers. At small apertures, diffraction and interference distort the autocollimator's reticle image on its CCD detector [72, 73]. Furthermore, the pixelation of the reticle image on the CCD detector, as well as inter- and intra-pixel variations in properties such as quantum efficiency, are interacting with the algorithms which are used for the sub-pixel interpolation of the location of the image on the CCD. This interaction leads to angle measurement errors with a period which corresponds to the CCD pixel size and which are hard to characterise as the autocollimator calibration needs to be performed with high angular resolution across the entire measurement range [53, 69]. As far as the CCD detector is concerned, the majority of its characteristics (such as variations between pixels in quantum efficiency, dark current, charge transfer efficiency, amplifier gain, and bias) can be calibrated and corrected rather easily [74-76]. However, characteristics such as errors in the CCD's pixel position due to chip fabrication tolerances or intra-pixel quantum efficiency variations (i.e., changes in the light sensitivity across each pixel due to its internal make-up [77, 78]) resist easy calibration.

In cooperation with the autocollimator manufacturer Möller-Wedel Optical (MWO) [79], Germany, PTB will address issues such as the adaptation of the reticle pattern which is imaged on the CCD to the CCD's specific properties and the improvement of the algorithms used for the sub-pixel location of the reticle image on the CCD by use of an Elcomat Direct [80]. This work is in progress.

\subsection{Application of autocollimators in profilometry}

Influences of the measuring conditions on the autocollimator's angle response are of special importance to its application in deflectometric profilometers for the precision form measurement of optics. A special - but not exclusive - focus was set on the form measurement of beam-guiding and -shaping optics for synchrotron and FEL beamlines which relies on autocollimator-based profilometers as these applications are the most challenging ones (due to the size, strong curvature, and stringent shape tolerances of the measured optics). Other applications include the measurement of reference surfaces for traceable interferometer calibration at National Metrology Institutes (NMI). This Work Package focuses on the influences of properties of the SUT, such as its curvature and reflectivity, and on the highly reproducible positioning of the aperture which, in profilometers, defines the beam footprint on the SUT. More details are provided in a review of the 
state-of-the-art of angle-based precision form measurement of optical surfaces with deflectometric profilometers which can be accessed via [20].

One focus is on characterising the influences of properties of the SUT, primarily its reflectivity and curvature, on the angle response of autocollimators. Most synchrotron and FEL beamline optics feature strong and locally varying curvatures which affect both the location and the quality of the image of the autocollimator's reticle on the CCD detector. Beamline optics even exhibit different radii of curvature in longitudinal and sagittal directions. The influences of these surface characteristics have not yet been investigated in detail and a systematic effort to characterise them is essential for advancing deflectometric form measurement. To achieve these goals, extensive experimental characterisation of the influence of flatness deviations of the SUT on the autocollimator's angle measurement by using surfaces of different radii of curvature and different beam path lengths will be performed. Ray tracing modelling of the curvature's influence on the autocollimator's angle response will be used to support the experimental work. Experimental work also includes the characterisation of the influence of the reflectivity of the SUT on the autocollimator's angle measurement. In this case, ray tracing simulations are not possible as stray light influences cannot be modelled adequately.

In deflectometric profilometers, a circular aperture is used to restrict the beam footprint on the SUT. It's centring is a crucial task. Therefore, we are developing a device and a standardised procedure for the highly reproducible $(<0.1 \mathrm{~mm})$ positioning of small $(1.5-2.5 \mathrm{~mm})$ apertures near the SUT relative to the autocollimator's optical axis during autocollimator use and calibration. This development is especially relevant to the application of autocollimators in deflectometric profilometers for the precision form measurement of beam-shaping optical surfaces for synchrotrons and FEL $[43,81,82]$. As smaller apertures are necessary to improve the lateral resolution in profilometry and demands on synchrotron and FEL optics are increasing, the requirements for aperture positioning get ever more stringent. The goal is to achieve a reproducibility of the centring of $<0.1 \mathrm{~mm}$. This research is crucial for traceable angle metrology with autocollimators as their angle response depends sensitively on their measuring conditions (including the aperture's position [69]).

The autocollimator manufacturer MWO [79] is currently creating an aperture centring device which can be attached to its Elcomat 3000 series of autocollimators [83]. PTB did provide ray tracing simulations of an autocollimator with variable aperture positions. Subsequently, the capabilities of the device and procedure will be verified experimentally by its application at different NMIs and synchrotron and FEL metrology laboratories and by intercomparison of autocollimator calibrations performed with the device.

\section{IMPROVING THE ASSURANCE OF TRACEABILITY IN ADVANCED ANGLE METROLOGY}

The aim of the Work Packages 'Metrological investigations on precise angle encoders' and 'Small angle generators and hybrid angle comparators' of project SIB58 Angles is to significantly improve angle metrology for dissemination of the SI unit 'radian' realised using 'subdivision of full circle $-2 \pi$ rad' and 'ratio of two lengths' respectively. The specific aim is to improve the performance of precise angle encoders and small angle generators for assurance of traceability with the uncertainties better than 0.01 " $(50 \mathrm{nrad})$ that is required particularly for calibration of autocollimators. This will be achieved performing investigations on extensive experimental characterisation and calibration of these devices using various approaches, new emerging methods and developments. The results aim at creating significant advances in generation of small angles at nanoradian (nrad) uncertainty and sub-nanoradian sensitivity values that will be used for traceability issues.

\subsection{Metrological investigations on precise angle encoders}

As defined in section 2-4, very low uncertainty demands arise for small angle measurements in order to provide traceability during investigations and enhancement of autocollimators' performance under extremely challenging measuring conditions. In order to tackle such challenging work adequately, this Work Package focuses on the development of novel concept rotary tables (based on a rotating encoder using a pair of reading heads), investigations on calibration of rotary tables fitted with multiple and one reading head angle encoders, novel methods (e.g. shearing techniques) for autocollimator calibration using angle encoders and new emerging methods for better signal interpolations in angle encoders in order to achieve high level precision and uncertainties better than current state of the art uncertainty values (e.g. $\mathrm{U}=0.01$ "). 
A new rotary table (so called Rotating Encoder Standard, RES) is under development in INRIM aiming to reduce the non-uniformity and interpolation errors by applying a concept of using a pair of heads, one fixed and a second rotating with the measurement drum.

Investigations on calibration of rotary tables fitted with 'multiple reading head' angle encoders have been started in PTB and a self-calibration method for the fast and precise in-situ calibration of multiple head angle encoders without recourse to external reference standards has been developed $[20,84]$. The method relies on a suitable geometric arrangement of multiple reading heads, which read out the radial grating of the angle encoder at different angular positions. Fourierbased algorithms are used to analyse the measurement differences of pairs of heads to recover the graduation error of the grating. The evaluation and correction of error influences due to lateral shifts of the centre of the encoder's grating during its rotation have also been achieved. Detailed information is available in [20, 84].

Investigations to develop novel method for precise calibration of angle encoders with 'one reading head' using the supplementary correction data to achieve required uncertainties less than 0.01 " $(\mathrm{k}=2)$ is in progress. Extensive experimental characterisation and calibration of one reading head encoders are being carried out using various approaches in TUBITAK, CEM, FAGOR AUTOMATION, MG, AIST and LNE. The approaches include investigation of form errors against errors of angle encoder and their effects, calibration of angle encoders using available methods but further correcting with supplementary correction data, investigations in encoder calibration using another encoder with controlled and measured alignment methods using special set developed and investigations on calibration of encoders using self-calibration and comparison versus a second commercially available encoder taken as a standard.

The application of advanced error-separating shearing techniques to the precise calibration of autocollimators with angle encoders and thereby to improve the classical calibration methods to reach lower expanded uncertainties (about 0.005 ") is in progress. Most NMIs use rotary tables fitted with angle encoders for the realisation of SI unit 'radian' to provide traceability in autocollimator calibrations. However, prior to autocollimator calibration, the performance of the angle encoder in the rotary table needs to be characterised by use of various methods (e.g., self- and cross-calibration) limiting the uncertainty values which can be achieved in autocollimator calibration. In contrast, shearing techniques, by applying defined angle offsets between both systems, offer a unique opportunity to separate the errors of the autocollimator and of the angle encoder and, therefore, to calibrate both systems without recourse to any external standard. Therefore, by error separation, shearing techniques provide simultaneous access to the angle deviations of the autocollimator and to those of the angle encoder in the rotary table and thus allow reducing the uncertainty of the angle measurement with both systems.

The adaptation of advanced error-separating shearing techniques to the precise calibration of autocollimators with angle encoders has been accomplished and demonstrated experimentally in PTB [20,85]. Autocollimator and angle encoder errors were separated with very small residuals demonstrating systematic error influences at a level below 2 nrad (0.0004"). This achievement is impressive and provides a solid base for improvement of the classical autocollimator calibration methods to reach substantially lower calibration uncertainties. Detailed information is available in [20, 85].

The shearing method has been demonstrated experimentally in clean room environment using the special angle comparator of PTB (WMT 220). It has been shown that error-separation with a standard measurement uncertainty at a level of 1 miliarcsec $(5 \mathrm{nrad})$ which beats the uncertainties reachable by conventional calibration methods for autocollimators by a factor of two to three $[20,85]$. The shearing method merely assumes that three sets of calibration data (obtained by applying defined angle offsets between autocollimator and the angle encoder) are reproducible, i.e., two signals generated from three shearing data sets are not changed by introducing phase shifts between them. This indeed requires reproducible environment conditions and reference angle measurement devices. Now the work is in progress to apply this method to various angle measurement devices (different Rotary Tables fitted with different angle encoders and head arrangements) of consortium members in project SIB58 Angles (TUBITAK, INRIM, MG, CEM, LNE, AIST and IPQ). The aim is to evaluate the results obtained from different types of rotary tables fitted with different angle encoders for application of shearing methods.

New methods for better signal interpolations in angle encoders are in progress. The aim is to develop improved methods for signal interpolation (between graduations of the grating, i.e. lines) of the encoder's reading heads (one head and multiple heads) with a focus on the determination and correction of interpolation errors. Shearing methods will be utilised here by applying to the different angle encoders of TUBITAK, INRIM, MG, CEM, FAGOR AUTOMATION for single reading heads and of AIST and PTB for multiple reading heads. First results taken in PTB [85] showed that shearing method is ideally suited for the calibration of interpolation errors of the devices at small angular scales which 
are difficult to characterise with other methods. Development of new configuration for image scanning type encoders and theoretical analysis is carried out in KRISS.

\subsection{Small angle generators and hybrid angle comparators}

In order to provide generated small angles for extremely challenging values such as nanoradian (nrad) uncertainty and sub-nanoradian sensitivity, this Work Package performs extensive research work and new developments for improvement of small angle generating and hybrid devices. This includes investigations on nanoradian uncertainty and sub-nanoradian sensitivity angle generation and measurements, improvements in current available methods and application of angle measurement that will be done using Differential Fabry-Perot interferometer and frequency stabilised lasers providing displacement measurements at picometre sensitivity free from linearity errors, development of portable small angle generator for calibration of autocollimators with an uncertainty of 0.01 ", and investigations and further improvements in hybrid angle comparators. Applications of advanced error-separating shearing techniques to Small Angle Generators (SAGs) for calibration of precise autocollimators are also being carried out to further investigate the errors sources since SAGs will show different systematic errors than angle encoders.

Extensive experimental investigations using current state of the art high precision small angle generators (i.e. nano angle generators) [56, 61] focusing on the capability of available devices and improvement on ambient conditions are in progress at TUBITAK and INRIM. It is aimed to search the possibilities for reduction of 2 nrad repeatability to sub nanorad level. Parameters in the uncertainty budget are being investigated for improvement. In addition, angle measurement using Differential Fabry-Perot interferometer and frequency stabilised lasers [86] are in progress at TUBITAK. Two laser heads are configured in various distances to each other (approx. $40-100 \mathrm{~mm}$ ) to measure the small angles using definition of SI unit radian principle. The aim here is to utilise the picometre level sensitivity (with linearity error free) displacement measurement system for generation of angles in sub-nanoradian sensitivity.

Three different Large Range Small Angle Generators (LRSAGs) are in the process of development by involvement of TUBITAK, INRIM, PTB, CEM, IK4-TEKNIKER, SMD, CMI and HZB according to requirements in section 3. Different mechanisms for small angle generation are being developed and different angle measurement scales for these systems such as calibrated reference autocollimators, Fizeau Angle Interferometer (FAI), conventional angle Interferometers based on displacement measurements with different configuration and data processing are being investigated for integration to the developed mechanisms.

Application of novel calibration methods for autocollimators using Small Angle Generators (SAGs) are in progress. The aim is to investigate error sources of SAGs more precisely utilising advanced error-separating shearing techniques for calibration of precise autocollimators. Developed shearing method presented in section 5.1 will be adapted to calibration of autocollimators using SAGs of TUBITAK and INRIM.

Error sources of hybrid angle calibrators based on the integration of rotary tables fitted with angle encoder and angular interferometer for generation of small angles are being investigated. The target uncertainty is of from $\mathrm{u} \leq 0,001$ arcsec for angles up to 1 degree till $\mathrm{u} \leq 0,005$ arcsec for angles up to10 degrees.

\section{CONCLUSIONS}

Joint Research Project (JRP) SIB58 Angles of the European Metrology Research Programme (EMRP) was presented and highlights summarising its progress with the first results were reported. The community of metrology for X-Ray and EUV Optics were informed about the latest work in angle metrology that will be a use for the development of SR optics required for beamlines at the next generation of synchrotron sources like Free Electron Lasers (FEL) and Diffraction Limited Storage Rings (DLSR).

It was shown that JRP aimed to give more weight to stakeholder needs with respect to the improved dissemination of the SI angle unit 'radian', which was currently demanded by the most challenging applications at the forefront of angle metrology and a range of precise angle measuring devices, applications and challenging measuring conditions were addressed in the project.

The first results showed that shearing techniques, offered a unique opportunity to separate the errors of the test and reference measurement system and hence, to calibrate both systems without recourse to any external standard. The adaptation of advanced error-separating shearing techniques to the precise calibration of autocollimators with angle encoders has been accomplished and demonstrated experimentally in PTB. Autocollimator and angle encoder errors 
were separated with very small residuals demonstrating systematic error influences at a level below 2 nrad (0.0004"). This achievement was impressive and provided a solid base for improvement of the classical autocollimator calibration methods to reach substantially lower calibration uncertainties. It was reported that error-separation with a standard measurement uncertainty at a level of 1 miliarcsec $(5 \mathrm{nrad})$ which beats the uncertainties reachable by conventional calibration methods for autocollimators by a factor of two to three was achieved. The work is in progress for application of shearing method to calibration of autocollimators using different angle encoders (i.e. RTs) and small angle generators (SAGs) of consortium members in project SIB58 Angles.

Two different innovative set-ups for two-dimensional (2D) calibrations of autocollimators and investigations on applications on autocollimators in challenging conditions of profilometry such as small (mm-sized) apertures, a variable path length of the measuring beam, curved reflecting surfaces, and beam deflections in both orthogonal measuring axes have been started. The investigations on precise angle encoders (both, multiple and single reading head) have been started in order to define the error sources preventing precise applications. The specific needs of the end users, e.g. current and also future needs of accelerator based (ring- and linac-type) synchrotron facilities, have been determined in detail for calibration of autocollimators used in challenging conditions.

It was also presented that cooperative work of project SIB58 Angles brings the stakeholders together to provide solutions to the current problems by systematic approach. Development of aperture centring device by the autocollimator manufacturer MWO, (one of the project partners), according to defined parameters in the project (leaded by HZB and PTB) and its application at project partners (NMIs) and collaborators (synchrotron and FEL metrology laboratories) was given as an example.

Angle metrology is an enabling technology for a broad range of measuring and manufacturing equipment in almost all sectors of industry. It is considered that outcomes of the project will have high level impact and will be used mostly by community of metrology for X-Ray and EUV Optics.

\section{ACKNOWLEDGMENTS}

The authors acknowledge funding from project SIB58 Angles of the European Metrology Research Programme (EMRP). The EMRP is jointly funded by the EMRP participating countries within EURAMET and the European Union.

\section{REFERENCES}

[1] SI Brochure (8th Edit.), <http://www.bipm.org/en/si/> (27 June 2014). http://www.bipm.org/

[2] Evans, J. C. and Taylerson C. O., [Measurement of angle in engineering], National Physical Laboratory, London: HMSO, (1986).

[3] Sim, P. J., "Angle standards and their calibration," Modern Techniques in Metrology ed P L Hewitt (Singapore: World Scientific), 102-121 (1984).

[4] Kiyono, S., Zhang S., and Uda Y., "Self-calibration of precision angle sensor and polygon mirror," Measurement 21(4), 125-136 (1997).

[5] Estler, W. T., "Uncertainty analysis for angle calibrations using circle closure," J. Res. Natl. Inst. Stand. Technol. 103, 141151 (1998).

[6] Yandayan T., Akgöz S. A. and Haitjema H., "A novel technique for calibration of polygon angles with noninteger subdivision of indexing table," Precision Engineering: J. Int. Soc. Precision Engineering and Nanotechnology 26, 412-424 (2002).

[7] Estler, W. T. and Queen, Y. H., “An advanced angle metrology system,” Ann. CIRP 42(1), 573576 (1993).

[8] Probst R., Wittekopf R., Krause M., Dangschat H. and Ernst A., "The new PTB angle comparator," Meas. Sci. Technol. 9, 1059-1066 (1998).

[9] Masuda T. and Kajitani M., “An automatic calibration system for angular encoders,” Precis. Eng. 11, 95-100 (1989).

[10] Masuda T. and Kajitani M., "High accuracy calibration system for angular encoders,” J. Robot. Mechatron. 5, 448-452 (1993). 
[11] Just A., Krause M., Probst R., and Wittekopt R., "Calibration of high-resolution electronic autocollimators against an angle comparator," Metrologia 40, 288-294 (2003)

[12] Watanabe T., Fujimoto H., Nakayama K., Masuda T. and Kajitani M., “Automatic high precision calibration system for angle encoder," Proc. SPIE 4401 267-274 (2001).

[13] Watanabe T., Fujimoto H., Nakayama K., Masuda T. and Kajitani M., "Automatic high precision calibration system for angle encoder II," Proc. SPIE 5190, 400-409 (2003).

[14] Watanabe T., Fujimoto H., Nakayama K., Kajitani M. and Masuda T., "Calibration of a polygon mirror by the rotary encoder calibration system," Proc. 17th IMEKO World Congress, 1890-1893 (2003).

[15] Yandayan T., "Application of the novel technique for calibration of 23-sided polygon with non-integer subdivision of indexing table," VDI/VDE-Gesellschaft Mess- und Automatisierungstechnik VDI-Berichte 1860; Measurement and Quality Control in Production; Symposium Erlangen VDI-Verlag Duesseldorf, 769-774 (2004).

[16] The Metre Convention, <http:/www.bipm.org/en/convention/> (27 June 2014). http://www.bipm.org/

[17] EURAMET e.V. <http://www.euramet.org/index.php?id=homepage > (27 June 2014).

[18] Research EMRP, < http://www.euramet.org/index.php?id=about_emrp>, (27 June 2014).

[19]Publishable JRP Summary Report for JRP SIB58 Angles, Angle Metrology, $<$ http://www.euramet.org/fileadmin/docs/EMRP/JRP/JRP_Summaries_2012/SI_Broader_Scope_JRPs/SIB58_P ublishable_JRP_Summary.pdf $>$ (27 June 2014).

[20] JRP SIB58 Angles, Angle Metrology website, < http://www.anglemetrology.com/>, (27 June 2014).

[21]Debler E. and Zander K., "Ebenheitsmessung an optischen Planflächen mit Autokollimationsfernrohr und Pentagonprisma, PTB Mitteilungen Forschen + Prüfen, Amts und Mitteilungsblatt der Physikalisch Technischen Bundesanstalt, Braunschweig und Berlin," 339-349 (1979).

[22] Von Bieren K., "Pencil beam interferometer for aspheric optical surfaces," Proc. SPIE 343, 101-108 (1982).

[23] Takacs P., Qian S. N. and Colbert J., "Design of a long trace surface profiler," Proc. SPIE 749, 59-64 (1987).

[24] Gubarev M., Kester T. and Takacs P., "Calibration of a vertical-scan long trace profiler at MSFC, Optical Manufacturing and Testing IV", Proc. SPIE 4451 (2001).

[25] Takacs P. and Qian S. N., "Surface Profiling interferometer," US patent No.U4884697, (1989)

[26] Schulz M., Ehret G., Stavridis M. and Elster C., "Concept, design and capability analysis of the new Deflectometric Flatness Reference at PTB," Nucl. Instrum. Meth. A 616 (2010)

[27] Ehret G., Schulz M., Stavridis M., and Elster C., "Deflectometric systems for absolute flatness measurements at PTB," Meas. Sci. Technol. 23 (2012)

[28]Ehret G., Schulz M., Baier M. and Fitzenreiter A., "Optical measurement of absolute flatness with the deflectometric measurement systems at PTB,” J. Phys. 425 (2013).

[29] Siewert F., Noll T., Schlegel T., Zeschke T., and Lammert H., "The Nanometer Optical Component Measuring machine: a new Sub-nm Topography Measuring Device for X-ray Optics at BESSY," AIP Conference Proceedings 705, American Institute of Physics, 847-850 (2004).

[30]Lammert H., Noll T., Schlegel T., Siewert F. and Zeschke T., "Optisches Messverfahren und Präzisionsmessmaschine zur Ermittlung von Idealformabweichungen technisch polierter Oberflächen," Patent No.: DE 10303659 (2005).

[31] Irick S., "Improved measurement accuracy in a long trace profiler: compensation for laser pointing instability," Nucl. Instrum. Meth. A347, 226-230 (1994).

[32] Kirschman J.L., Domning E.E., McKinney W.R., Morrison G.Y., Smith B.V. and Yashchuk V.V., "Performance of the upgraded LTP-II at the ALS Optical Metrology Laboratory," Proc. SPIE 7077 (2008).

[33] Susini J., Baker R. and Vivo A., "Optical metrology facility at the ESRF," Rev. Sci. Instrum. 66 (2) (1995)

[34] Qian S., Werner J., and Takacs P., "The penta-prism LTP: A long-trace-profiler with stationary optical head and moving penta prism,” Rev. Sci. Instrum. 66 (3) (1995).

[35] Thomasset M., Brochet S. and Polack F., "Latest metrology results with the SOLEIL synchrotron LTP," Proc. SPIE 5921 (2005).

[36] Senba Y., Kishimoto H., Ohashi H., Yumoto H., Zeschke T., Siewert F., Goto S. and Ishikawa T., "Upgrade of long trace profiler for characterization of high-precision X-ray mirrors at SPring-8”, Nucl. Instrum. Meth. A 616, 237-240 (2010).

[37] Siewert F., Buchheim J. and Zeschke T., "Calibration and characterization of 2nd generation slope measuring profiler,” Nucl. Instrum. Meth. A 616, 119-127 (2010). 
[38] Alcock S.G., Sawhney K.J.S., Scott S., Pedersen U., Walton R., Siewert F., Zeschke T., Noll T. and Lammert H., "The Diamond-NOM: A non-contact profiler capable of characterizing optical figure error with sub-nm repeatability," Nucl. Instrum. Meth. A 616, 224-228 (2010).

[39] Nicolas J. and Martinez J. C., "Characterization of the error budget of the Alba-NOM," Nucl. Instrum. Meth. A 710, 24-30 (2013).

[40] Assoufid L., Brown N., Crews D., Sullivan J., Erdmann M., Qian J., Jemian P., Yashchuk V. V., Takacs P. Z., Artemiev N. A., Merthe D. J., McKinney W. R., Siewert F. and Zeschke T., "Development of a highperformance gantry system for a new generation of optical slope measuring profilers," Nucl. Instrum. Meth. A 710 (2013).

[41] Qian J., Sulivan J., Erdmann M., Khounsary A. and Assoufid L., "Performance of the APS optical slope measuring system," Nucl. Instrum. Meth. A 710 (2013).

[42] Idir M., Kaznatcheev K., Qian S.N. and Conley R., "Current status of the NSLS-II optical metrology laboratory," Nucl. Instrum. Meth. A 710 (2013).

[43] Yashchuk V.V., Barber S., Domning E.E., Kirschman J.L., Morrison G.Y., Smith B.V., Siewert F., Zeschke T., Geckeler R. and Just A., "Sub-microradian surface slope metrology with the ALS Developmental Long Trace Profiler," Nucl. Instrum. Meth. A 616 212-223 (2010).

[44] Altarelli M. et all. , "The European X-Ray Free Electron Laser - Technical Design Report,” DESY, Hamburg, (2006).

[45] Mimura H., Handa S., Kimura T., Yumoto H., Yamakawa D., Yokoyama H., Matsuyama S., Inagaki K., Yamamura K., Sano Y., Tamasaku K., Nishino Y., Yabash M. I, Ishikawa T. and Yamauchi K., "Breaking the $10 \mathrm{~nm}$ barrier in hard-X-ray focusing," Nature Physics, 1457 (2009).

[46] Rack A., Weitkamp T., Riotte M., Grigoriev D., Rack T., Helfen L., Baumbach T., Dietsch R., Holz T., Krämer M., Siewert F., Meduna M., Cloetens P. and Ziegler E., "Comparative study of multilayers used in monochromators for synchrotron-based coherent hard X-ray imaging,” J. Synchrotron Rad. 17 (2010).

[47] Rack A., Riesemeier H., Vagovic P., Weitkamp T., Siewert F., Dietsch R., Diete W., Bauer-Trabelsi S., Waterstradt T. and Baumbach T., "Fully automated, fixed exit, in vacuum double-multilayer monochromator for synchrotron-based hard Xray micro-imaging applications" AIP Conference Proc. 1234, 740-743, (2010)

[48] Irick S.C., Rev. Sci. Instrum 63(1) (1992).

[49] Signorato R. and Sanchez del Rio M., "Structured slope errors on real x-ray mirrors: Ray tracing versus Experiment," Proc. SPIE 3152, 136-147 (1997).

[50]F. Siewert, [Slope Error and Surface Roughness, in: Modern Developments in X-ray and Neutron Optics], Springer, Berlin Heidelberg, 175-179 (2008).

[51] Ehret G., Schulz M., Stavridis M. and Elster C., "A new flatness reference measurement system based on deflectometry and difference defelctometry," in Fringe 2009: 6th International Workshop on Advanced Optical Metrology, W. Osten, M. Kujawinska eds, Springer-Verlag, Berlin, 318-323 (2009).

[52] Yashchuk V., McKinney W., Warwick T., Noll T., Siewert F., Zeschke T. and Geckeler R., "Proposal for a Universal Test Mirror for Characterization of Slope Measuring Instruments,” Proc. SPIE, 6704, (2007).

[53] Geckeler R.D., Just A., Krause M. and Yashchuk V.V., "Autocollimators for deflectometry: Current status and future progress,” Nucl. Instrum. Meth. A 616, 140-146 (2010).

[54] Kren P., "A simple interferometric method for determining the flatness of large optical flats with $1 \mathrm{~nm}$ repeatability," Meas. Sci. Technol. 19, 107001, 5pp (2008).

[55] Kren P, "Optical mirror referenced capacitive flatness measurement and straightness evaluation of translation stages," Meas. Sci. Technol. 25, 044017, 5pp (2014).

[56] Yandayan T, Ozgur B, Karaboce N, and Yaman O, "High precision small angle generator for realization of the SI unit of plane angle and calibration of high precision autocollimators" Meas. Sci. Technol. 23, 094006, 12pp. (2012).

[57] Yandayan T, Akgoz S A, and Asar M, Calibration of high-resolution electronic autocollimators with demanded low uncertainties using single reading head angle encoders" Meas. Sci. Technol. 25, 015010, 16pp (2014).

[58] Autocollimators, $\quad<$ http://www.moeller-wedel-optical.com/en/produkte/electronic-autocollimators.html $>$ (27 June 2014).

[59] Yandayan T., Karaboce N., Ozgur B. and Yaman O. "Small angle generators for angle measurement," International Metrology Conference CAFMET, (2010)

[60] CMC (Calibration Measurement Capabilities) database of the BIPM, <http://kcdb.bipm.org/appendixC/> (27 June 2014). 
[61] Astrua M. and Pisani M. “The new INRiM nanoangle generator,” Metrologia 46, 674-81 (2009).

[62] METAS website, <http://www.metas.ch/metasweb/Fachbereiche/Laenge/Winkel> (27 June 2014).

[63] Geckeler R. D. and Just A. "Angle comparison using an autocollimator EURAMET.L-K3.2009 Technical Protocol, EURAMET Project No 1074,” <http://kcdb.bipm.org/AppendixB/appbresults/EURAMET.LK3.2009/EURAMET.L-K3.2009_Techical_Protocol.pdf $>$ (27 June 2014).

[64] Samoylova L., Sinn H., Siewert F., Mimura H.,Yamauchi K. and T. Tschentscher, "Requirements on Hard Xray Grazing Incidence optics for European XFEL: Analysis and Simulation of Wavefront Transformations,", Proc. SPIE 7360 (2009).

[65] Siewert F., Buchheim J., Boutet S., Williams G.J., Montanez P.A., Krzywinski J. and Signorato R., "Ultraprecise characterization of LCLS hard X-ray focusing mirrors by high resolution slope measuring deflectometry," Optics Express 20, 4525-4536 (2012).

[66] Geckeler R. D., Weingartner I., Just A. and Probst R., "Use and traceable calibration of autocollimators for ultra-precise measurement of slope and topogragphy," Proc. SPIE 4401, 184-195 (2001).

[67] Geckeler R. D., "Error minimization in high-accuracy scanning deflectometry,” Proc. SPIE 6293, 1-12 (2006).

[68] Geckeler R. D. and Just A., "Distance dependent influences on angle metrology with autocollimators in deflectometry," Proc. SPIE 7077, 70770B 1-12 (2008).

[69] Geckeler R. D. and Just A., "Optimized use and calibration of autocollimators in deflectometry," Proc. SPIE 6704, 670407 1-12 (2007).

[70] Geckeler R. D., Kranz O., Just A., and Krause M., “A novel approach for extending autocollimator calibration from plane to spatial angles," Advanced Optical Technologies 1(6), 427-39 (2012).

[71] Kranz O., Geckeler R. D., Just A. and Krause M., "Modelling PTB's spatial angle autocollimator calibrator" Proc. SPIE 8789, 87890D 1-11 (2013).

[72] Fütterer G., "Simulation of the detectors response of an autocollimator," Proc. SPIE 6617, 661703 1-8 (2007).

[73] Fütterer G., "Enhancement of high resolution electronic autocollimators by application of phase grating technology," Proc. SPIE 5856, 950-959 (2005).

[74] Kirschman J. L., Smith B.V., Domning E. E., Irick S. C., MacDowell A. A., McKinney W. R., Morrison G. Y., Smith B. V., Warwick T. and Yashchuk V. V., "Flat-field calibration of CCD detector for Long Trace Profilers" Proc. SPIE 6317, 67040J 1-11 (2007).

[75] Yashchuk V. V., "Positioning errors of pencil-beam interferometers for long-trace profilers," Proc. SPIE 6317 63170A 1-12 (2006).

[76] Kavaldjiev D. and Ninkov Z., "Influence of non-uniform charge-coupled device pixel response on aperture photometry" Opt. Eng. 40(2) 162-169 (2001).

[77] Piterman A. and Ninkov Z., "Subpixel sensitivity maps for a back-illuminated charge-coupled device and the effects of nonuniform response on measurement accuracy," Opt. Eng. 41(6) 1192-1202 (2002).

[78] Kavaldjiev D. and Ninkov Z., "Subpixel sensitivity map for a charge-coupled device sensor,” Opt. Eng. 37(3) 948-954 (1998)

[79] Möller-Wedel Optical Website <http://www.moeller-wedel-optical.com/en.html> (1 July 2014).

[80]Elcomat Direct <http://www.moeller-wedel-optical.com/en/produkte/electronic-autocollimators/p-elcomatdirect.html $>$ (1 July 2014).

[81] Barber S. K., Geckeler R. D., Yashchuk V. V., Gubarev M. V., Buchheim J., Siewert F. and Zeschke T. "Optimally aligned mirror based pentaprism for scanning deflectometric devices," Opt. Eng. 50(7) 073602 1-8 (2011)

[82] Ehret G., Schulz M., Fitzenreiter A., Baier M., Jockel W., Stavridis M., and Elster C., “Alignment methods for ultraprecise deflectometric flatness metrology," Proc. SPIE 8082, 808213 1-8 (2011).

[83] ELCOMAT 3000, <http://www.moeller-wedel-optical.com/en/produkte/electronic-autocollimators/p-elcomat3000.html $>$ (1 July 2014).

[84] Geckeler R.D., Link A, Krause M, and Elster C., "Capabilities and limitations of the self-calibration of angle encoders," Meas. Sci. Technol. 25, 055003 10pp (2014).

[85] Geckeler R. D. and Just A. "A shearing-based method for the simultaneous calibration of angle measuring devices," To be published (2014).

[86] Çelik M., Hamid R., Kuetgens U., and Yacoot A., "Picometre displacement measurements using a differential Fabry-Perot optical interferometer and x-ray interferometer,” Meas. Sci. Technol. 23, 085901 6pp (2012). 